\title{
A study of the effects of fluoridation in Brazil
}

\author{
Prevalence of caries and developmental defects of enamel in 9-10 year old children living in areas in Brazil with \\ differing waterfluoride histories by E. L. Dini, R. D. Holt, and R. Bedi BrDent J 2000; 188: 146-149
}

\begin{abstract}
Aims
To assess the prevalences of caries, of developmental defects of enamel and their interrelationship in Brazilian 9-10-year-olds from areas of contrasting fluoridation histories.
\end{abstract}

\section{Methods}

Systematic random sampling procedures were used to select children from an area where water had been fluoridated in 1963 and from a second area where water had been fluoridated since 1998. Clinical examinations for caries were carried out using the DMFT index and WHO diagnostic criteria. Developmental defects of enamel on upper incisors were diagnosed using the DDE index.

\section{Results}

A difference of $40 \%$ in DMFT was observed, with a lower prevalence of disease in the area fluoridated since 1963. Diffuse opacities affected $14.3 \%$ of the children from the area fluoridated since 1963 compared with only $2.4 \%$ in the area fluoridated in 1998. Children living in the area fluoridated in 1963 who had diffuse defects had twice the chance of being free from caries compared with those living in the same area who had no defects or who had only demarcated or hypoplastic defects.

\section{Conclusions}

This study confirms previous ones in showing the benefits of water fluoridation. Diffuse opacities of upper incisors affected relatively few subjects in either of the two areas.

\section{In brief}

- Fluoridation in Araraquara, Brazil, has resulted in benefits equivalent to those seen in other parts of the world.

- The prevalence of diffuse opacities was low in areas with and without long-term fluoridation.

- In the fluoridated area of Araraquara, subjects with diffuse opacities had twice the chance of being free of caries.

\section{Comment}

This study compared children aged 9 to 10 years who had lived continuously in the city of Araraquara in Sao Paulo State, Brazil, where the water has contained $0.7 \mathrm{ppm}$ fluoride since 1963, and children living in the city of Americo Brasliense, where the water supply had been fluoridated for only a few months. The study had three aims:

- To compare caries experience and prevalence in the two communities

- To compare the prevalence of developmental defects of enamel

- To study the association, if any, between caries and diffuse enamel defects (dental fluorosis).

Cross-sectional comparisons of dental caries in fluoridated and non (or low) fluoridated communities such as these that have been reported in more than one hundred studies, (including Brazil), and further studies probably add little if anything to our knowledge.

There are countless numbers of such comparisons that could be made, and to avoid charges of selection bias it is necessary for authors to establish that their particular choices of fluoridated and non-fluoridated communities are comparable. The most convincing way of achieving this is to record pre-fluoridation baseline levels of dental caries in both communities. Indeed the current review of the effectiveness of water fluoridation conducted by the Centre for Reviews and Dissemination at the University of York will, in considering a casual relationship, only include prospective studies incorporating two or more comparison groups with data indicating their comparability at baseline. (http://www.york.ac.uk/ inst/crd/fluorid.htm)

Interestingly, the dental fluorosis data presented by the authors would be accepted by the York review team since they have set lower inclusion standards for studies of negative effects.

The most interesting aspect of this study relates to the authors' third aim; ie the association between dental fluorosis (diffuse defects) and caries prevalence. In the community, fluoridated since 1963 children with dental fluorosis on their central incisors are significantly less likely to have dental caries than children with no fluorosis. Because most of this dental caries is limited to permanent first molars this 'biologically based' aim would have been better investigated by also measuring fluorisis on the first permanent molars. This would have allowed a more in depth analysis of the relationship between caries and fluorosis at the level of the single site or tooth.

The biological plausibility of this interesting association has been discussed at some length by Ellwood and O'Mullane and is worthy of further research. ${ }^{1}$

\section{A Lennon}

Professor of Dental Public Health, Department of Clinical Dental Sciences, The University of Liverpool

1 Ellwood R P, O'Mullane D. The association between developmental enamel defects and caries in populations with and without fluoride in their drinking water. $J$ Public Health Dent 1996; 56: 76-80. 